\section{Turning the Tide of Data Sharing}

\author{
Giorgio A. Ascoli ${ }^{1,2,3,4}$
}

Published online: 14 October 2019

(C) Springer Science+Business Media, LLC, part of Springer Nature 2019

For the neuroscience ecosystem surrounding digital reconstructions of neuronal morphology, I predict that the past year will be remembered as a glorious time. The centrally curated community repository NeuroMorpho. Org $^{1}$ passed the landmark quota of 100,000 downloadable tracings in version 7.6 (end of November 2018), more than doubling from the $50 \mathrm{k}$ milestone reached only 24 months before. ${ }^{2}$ Considering that this database launched in 2006 with (what then appeared the impressive number of) 1000 neurons, ${ }^{3}$ and that it took all of seven years to add the next 9000 , the acceleration is impressive (Fig.1). The pivoting phase transition occurred around the second half of 2014: until then NeuroMorpho.Org averaged 1250 additional reconstructions per year; afterwards, roughly 20,000 . What triggered such a whopping 16-fold-factor?

A major reason is undoubtedly the industrialization of neuroscience, implying bigger and faster datasets. ${ }^{4}$ Experimental breakthroughs in imaging techniques, from the off-the-shelf availability of genetically labeled animals to the improving quality/cost ratio of microscopy, clearly played an important

\footnotetext{
${ }^{1}$ Akram, M.A., Nanda, S., Maraver, P., Armañanzas, R., Ascoli, G.A, (2018). An open repository for single-cell reconstructions of the brain forest. Scietific Data, 5, 180006. https://doi.org/10.1038/sdata.2018.6.

${ }^{2}$ Ascoli, G.A., Maraver, P., Nanda, S., Polavaram, S., Armañanzas, R. (2017). Win-win data sharing in neuroscience. Nature Methods, 14(2), 112-116.

${ }^{3}$ Ascoli, G.A., Donohue, D.E., Halavi, M. (2007). NeuroMorpho.Org: a central resource for neuronal morphologies. The Journal of Neuroscience, 27(35), 9247-51.

${ }^{4}$ Nanda, S., Allaham, M.M., Bergamino, M., Polavaram, S., Armañanzas, R., Ascoli, G.A., Parekh, R. (2015). Doubling up on the fly: NeuroMorpho.Org Meets Big Data. Neuroinformatics, 13(1), 127-9.
}

Giorgio A. Ascoli ascoli@gmu.edu

1 Bioengineering Department, George Mason University, Fairfax, VA, USA

2 Neuroscience Program, George Mason University, Fairfax, VA, USA

3 Center for Neural Informatics, Structures, \& Plasticity, George Mason University, Fairfax, VA, USA

4 Krasnow Institute for Advanced Study, George Mason University, Fairfax, VA, USA role. However, the lion's share of the merit for the rising production of neuronal reconstructions goes to informatics advances. The progressive computer automation of the difficult and tedious process of arbor tracing was a game changer for the field. ${ }^{5}$ As a result, the number of peer-reviewed publications reporting three-dimensional neural morphology increased from approximately 120 per year $^{6}$ before 2014 to over 300 after. Moreover, the average number of cells per publication also more than doubled in the same period, from just shy of 40 to nearly 100 . Thus, the yearly community-wide generation of reconstructed morphologies hovered below 5000 pre2014 and soared above 30,000 in the most recent few years.

Yet, ballooning data production by itself is insufficient to explain the tremendous growth of NeuroMorpho.Org. Another notable shift during this time regarded a fairly radical transformation in the neuroscientists' attitude towards data sharing. ${ }^{7}$ Up until 2014, three-quarters of the authors chose not to deposit the morphological reconstructions described in their publication into freely accessible storage. ${ }^{8}$ In the last five years, the wind has reversed, with two-thirds of researchers willingly uploading their hard-won tracings to the public domain cloud. Many interlinked factors likely contributed to this very encouraging trend: positive pressure from funding agencies and publishers, including the pioneering position of our journal $^{9}$; exemplary success stories of data reusage that amplified the impact of the original work ${ }^{10}$; greater name

\footnotetext{
${ }^{5}$ Peng, H., Meijering, E., Ascoli, G.A. (2015). From DIADEM to BigNeuron. Neuroinformatics, 13(3), 259-60.

${ }^{6}$ Halavi, M., Hamilton, K.A., Parekh, R., Ascoli, G.A. (2012). Digital reconstructions of neuronal morphology: three decades of research trends. Frontiers in Neuroscience, 6, 49. https://doi.org/10.3389/fnins.2012.00049

${ }^{7}$ Ascoli, G.A. (2015). Sharing Neuron Data: Carrots, Sticks, and Digital Records. PLoS Biology, 13(10), e1002275. https://doi.org/10.1371/journal. pbio. 1002275.

${ }^{8}$ Ascoli, G.A. (2006). The ups and downs of neuroscience shares. Neuroinformatics, 4(3), 213-6.

${ }^{9}$ Kennedy, D.N. (2017). The Information Sharing Statement Grows Some Teeth. Neuroinformatics, 15(2), 113-114.

${ }^{10}$ Ascoli, G.A. (2007). Successes and rewards in sharing digital reconstructions of neuronal morphology. Neuroinformatics, 5(3), 154-60.
} 
Fig. 1 Growth of

NeuroMorpho.Org content over

the 13-year period of this database existence. Two phases can be distinguished based on the rate of increase: the first 8 years $(\sim 1250$ reconstructions/year) and the last $5(\sim 20,000$ reconstructions per year)

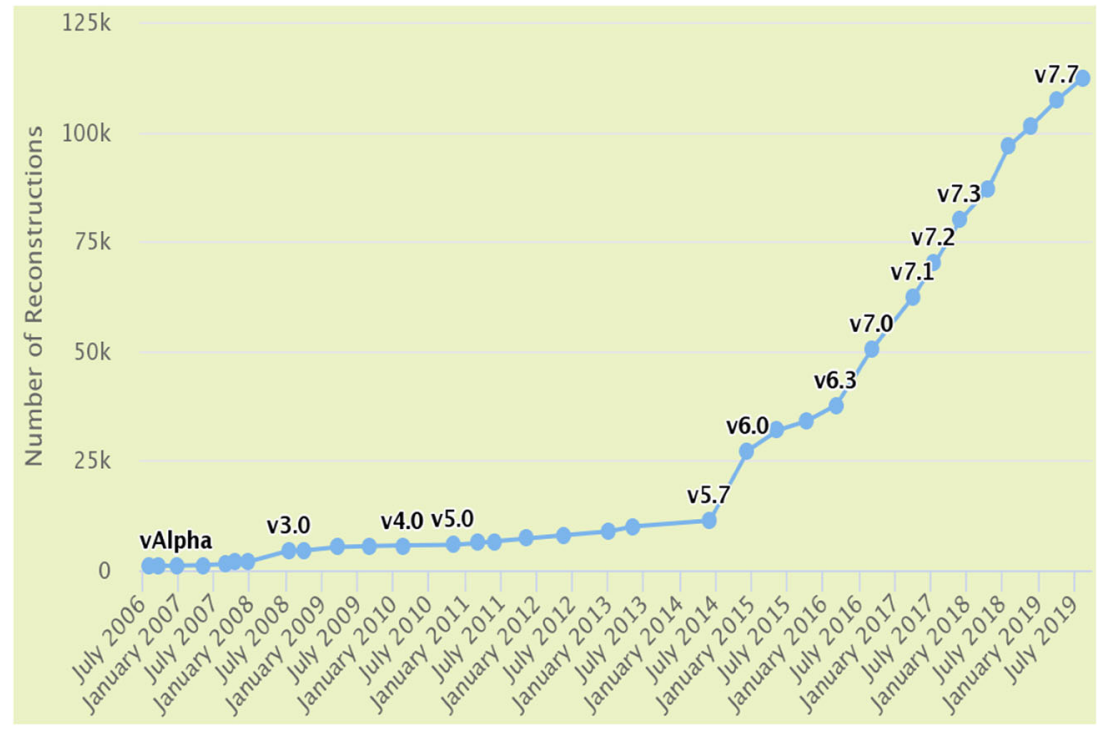

recognition and community trust in larger resources ${ }^{11}$; the growing prominence of data science; and a generational shift.

As a combined consequence of the data volume expansion and of the larger shared fraction, in January 2019 more than half of the digital neuronal morphologies ever reconstructed since the dawn of computerinterfaced microscopes (ca. 1985) were available through NeuroMorpho.Org. This is not a blip on the screen, but the new norm: seven months later, at the time of this writing, the number of shared morphological tracings exceed the un-shared pool by almost 15,000 , and the proportion of positive responses to the requests for data is edging towards $80 \%$. Meanwhile, technological progress continues in strides, and the "next" 100,000 neurons are already on the horizon. ${ }^{12}$ Furthermore, digital reconstructions no longer solely focus on the local dendritic field, but now encompass brain-wide axonal projections, ${ }^{13}$ with collaborative remote tracing enabled by immersive virtual reality environments. ${ }^{14}$

${ }^{11}$ Ascoli, G.A. (2014). A community spring for neuroscience data sharing. Neuroinformatics, 12(4), 509-11.

${ }^{12}$ DeWeerdt, S. (2019). How to map the brain. Nature Outlook 7/24/19. https://www.nature.com/articles/d41586-019-02208-0.

${ }^{13}$ Winnubst, J., Bas, E., Ferreira, T.A., Wu, Z., Economo, M.N., Edson, P., Arthur, B.J., Bruns, C., Rokicki, K., Schauder, D., Olbris, D.J., Murphy, S.D., Ackerman, D.G., Arshadi, C., Baldwin, P., Blake, R., Elsayed, A., Hasan, M., Ramirez, D., Dos Santos, B., Weldon, M., Zafar, A., Dudmann, J.T., Gerfen, C.R., Hantman, A.W., Korff, W., Sternson, S.M., Spruston, N., Svoboda, K., Chandrashekar, J. (2019). Reconstruction of 1000 projection neurons reveals new cell types and organization of long-range connectivity in the mouse brain. Cell SneakPeek. https://doi.org/10.1101/537233. http://papers.ssrn.com/sol3/ papers.cfm?abstract_id=3330557. BiorXiv.

${ }^{14}$ Wang, Y., Li, Q., Liu, L., Zhou, Z., Ruan, Z., Kong, L., Li, Y., Wang, Y., Zhong, N., Chai, R., Luo, X., Guo, Y., Hawrylycz, M., Luo, Q., Gu, Z., Xie, W., Zeng, H., Peng, H. (2019). TeraVR empowers precise reconstruction of complete 3-D neuronal morphology in the whole brain. Nature Communications, 10(1), 3474. https://doi.org/10.1038/s41467-019-11,443-y.
Perhaps most consequentially, new powerful synergies are emerging within the US BRAIN Initiative Cell Census Network (BICCN) as morphological reconstructions and single-neuron transcriptomics are paired within a common functional atlas. ${ }^{15}$ It is especially telling that the data produced under this 5-year, quarter-billion-dollar effort are intended for broad, rapid, online dissemination, and will become available for immediate and unrestricted use by the entire scientific community upon production and delivery to public databases (biccn.org). External users may freely download, analyze and publish results based on any BICCN open-access datasets and tools as soon as they are released, regardless of type or size and even if not yet published by the generating labs. Too good to be true? Maybe. Then again, in just a few years we might look back at 2019 trying to remember why 100,000 neurons seemed so many.

\section{Information Sharing Statement}

The data trends reported in this editorial are available at NeuroMorpho.Org from the "About", "What's New", "Detailed statistics", and "Literature coverage" pages.

Acknowledgments NeuroMorpho.Org is supported by NIH R01NS39600, R01NS86082, and U01MH114829.

Publisher's Note Springer Nature remains neutral with regard to jurisdictional claims in published maps and institutional affiliations.

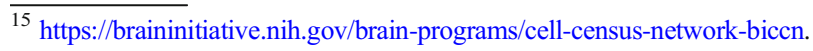

